\title{
Histochemistry of Equine Damaged Tendons, Ligaments and Articular Cartilage
}

\author{
Grasiela De Bastiani', Flávio Desessards De La Côrte², Karin Erica Brass², Camila Cantarelli², \\ Stefano Dau², Glaucia Denise Kommers' ${ }^{3}$, Taiara Müller Da Silva ${ }^{3}$ \& Marcos Da Silva Azevedo ${ }^{4}$
}

\begin{abstract}
Background: The injury repair process in tendons and ligaments includes different phases such as inflammation, neovascularization, fibroblast proliferation and fibrosis. Collagen type and tissue characteristics of tendon and ligament repair are described such as type collagen differentiation and properties of the scars tissue. The degeneration of articular cartilage when, characterized by loss of the articular layers associated of the decreased of proteoglycans. The aim of this study is to describe by histochemistry techniques the characteristics of tissue scar, collagen type in the repair process of tendons and ligaments, as well as articular cartilage degeneration.

Materials, Methods \& Results: Tissue samples of equine tendons, ligaments and articular cartilage of the metacarpophalangeal joint region were evaluated by ultrasonography, macroscopically and prepared for routine histopathology (H\&E staining). The inclusion criterion of the samples in this study was based on the presence of lesions characterized in $\mathrm{H} \& \mathrm{E}$ stain as fibroplasia, neovascularization, collagenolysis, chondroid metaplasia in tendons and ligaments and fibrillation and cartilaginous eburnation lesions in the articular cartilage samples. The Masson's trichrome, Picrosirius red and Alcian blue staining techniques were also performed in addition to H\&E. Pathologic findings in the tendons and ligaments included fibroplasia, collagenolysis, chondroid metaplasia and lymphohistioplasmacytic inflammation. Tendons and ligaments scars were composed of type III collagen but there was also some type I collagen. Fiber alignment of tendons and ligaments in the reorganization tissue was not flawless and the fiber appearance was characterized by a lack of the fiber crimp and parallelism. The fibroplasia was characterized by endotendinous tickening areas associated with the presence of loose connective tissue. In the areas of loose connective tissue substitution, collagen type fibers are intercalated to a lesser extent by type-III collagen fibers. In the Alcian blue stained samples of articular cartilage observed the surface layer and the matrix zone of calcified cartilage were weakly stained in blue.

Discussion: Three special stains were utilized in this study along with the H\&E evaluation elucidating the behavior tendons, ligaments and articular cartilage injury. The important observation in this study was fibroplasia in tendons and ligaments seems to be composed by abundant of loose connective tissue, chondrocytes and intermingled collagen type I and III fibers associated with lack of crimps alignment of the fibers. The fragile structure suggested by the Masson's trichrome stain results (presence of the loose connective tissue) in this study perhaps make the tendons and ligaments receptive to other lesions. The characteristic blue discoloration of collagen fibers was only observed in the loose connective tissue may be because the dye penetration becomes easier when compared to the dense connective tissue (stained in red). The Masson's trichrome made possible the differentiated the dense connective tissue of the loose connective tissue. The combined histochemistry staining technics allowed an improved characterization of fiber alignment, collagen type, inflammatory cell infiltration and neovascularization, which happens during the repair process of tendons and ligaments. The fibrillation and eburnation of the articular cartilage were associated with the decrease Alcian Blue staining characterized by degeneration process of articular cartilage.
\end{abstract}

Keywords: tendons, ligaments, articular cartilage, histochemistry.

DOI: $10.22456 / 1679-9216.88281$ 


\section{INTRODUCTION}

Mechanical stress capable of altering the blood supply of tendons and ligaments has been considered the primary cause of injuries [28], damaging the microvasculature as consequence of tendon overuse [12]. Therefore, soft tissue lesions are important for performance horses [21]. Tendon damage is also a common injury in racing Thoroughbreds and other competition horses and with many other species, leading to the formation of biomechanically weaker scar tissue and, therefore, resulting in a high probability of re-injury [8].

Fascicles of collagen are arranged in a waveform called crimp, and are responsible for promoting impact absorption that the tissue undergoes. Different types of collagen have different functions in the tendons and ligaments tissues. The type I, II and III collagen fibers are responsible for maintaining the tissue architecture and rigidity [25]. Tendon matrix is composed predominantly of type I collagen, with a small percentage of other collagens [13]. Many stain techniques have been developed for the collagen fibers study, the most of which is the Masson's trichrome stain but, this stain cannot differentiate the type of collagen fibers [17].

The articular cartilage is characterized by a flexible connective tissue composed primarily of water (68-85\% of the weight), fibrilar matrix containing type II collagen (10-20\%), proteoglycans (5-10\%) and chondrocytes [18].

The repair process of tendons and ligaments includes different phases such as inflammation, neovascularization, proliferation of granulation tissue and fibrosis. However, the articular cartilage repair does not occur after the injury, and is characterized by fibrillation and eburnation.

\section{MATERIALS AND METHODS}

\section{Tissue obtaining and preparation for analysis}

Fourteen samples of tendons, ligaments and articular cartilage of the metacarpophalangeal joint of the forelimb equine specimens, were evaluated macroscopically. This samples were part of previous ultrasound and macroscopic study [7]. Tissue samples were fixed in $10 \%$ buffered formalin solution for 14 days. Then, they were processed routinely for histopathology. The samples were then cut into $3 \mu \mathrm{m}$ histological sections and stained by hematoxylin and eosin (H\&E) method. The Masson's trichrome $(\mathrm{MT})^{1}$, Picrosirius red $(\mathrm{PR})^{1}$ and Alcian blue (AB) staining techniques were also performed. The MT allows the collagen differentiation between the loose and the dense tissue in tendons and ligaments. The loose connective tissue stains in blue and the dense connective tissue stains in red. The PR was used for the differentiation of type I and III collagen fibers in tendons and ligaments, under polarized light. In PR stain, collagen type I fibers were orange and thick, differing from the collagen fibers type III, that were greenish to blue and thin. The articular cartilage sections (and the subchondral bone tissue), after fixation, underwent a decalcification process (with aquous solution of sodium citrate and formic acid). The samples were then processed routinely for histopathology. The $A B$ technique $(\mathrm{pH} 2,5)$ for identification of hyaline cartilage was modified [6] counterstained with $\mathrm{H} \& \mathrm{E}$. The AB stain demonstrated the presence of a myxoid-like matrix in fibroblastic tissue of tendons and ligments, that was AB-positive matrix. In samples of the articular cartilages, the $\mathrm{AB}$ stain demonstrated the hyaline chondoid matrix.

\section{Inclusion criterion of the samples}

The inclusion criterion of the samples in this study was based on the presence of lesions characterized in H\&E stain as fibroplasia, neovascularization, collagenolysis, chondroid metaplasia in tendons and ligaments and fibrillation and cartilaginous eburnation lesions in articular cartilage samples. Findings were divided according to the type of lesion observed in $\mathrm{H} \& \mathrm{E}$ study, divided into categories such as junction of Superficial Digital Flexor Tendon (SDFT) and Manica Flexoria (MF) (2), SDFT (1), Deep Digital Flexor Tendon (DDFT) (5), Palmar Annular Ligament (PAL) (2) of metacarpophalangeal joint palmar region, Suspensory Ligament (SL) lateral branch (1), and Articular Cartilage (AC) of metacarpus III (MCII) condyle, (3).

\section{RESULTS}

\section{Deep Digital Flexor Tendon (DDFT)}

Gross lesions of the DDFT in the cross section were characterized by hemorrhagic infiltration and red to dark-yellow zones and, in one sample, the consistency of the tendon was very soft.

The H\&E findings of the deep digital flexor tendon tissue of the palmar aspect of the metacarpophalangeal joint were divided into: fibroplasia (Figure 1-A), collagenolysis, neovascularization and chondroid metaplasia. 
The compact alignment of the collagen fibers and the dense connective tissue possibly make the penetration of the MT stained difficult and reproduce the red color stain. In contrast, the loose connective tissue presented blue tinctorial property and the penetration of the TM stain was easy by done.

In the MT-stained samples, fibroplasia (Figure $1-\mathrm{C}$ ) in the DDFT was characterized by endotendinous thickening areas with the presence of generous amount of loose connective tissue. In these areas, the dense connective tissue was replaced by the loose connective tissue. These same regions in AB (Figure 1-B) staining presented as a myxoid-lyke matrix. In the PR-stained samples, the dense connective tissue corresponded of thick orange collagen fibers [type-I collagen fibers] (Figure 1-C). The loose connective tissue is composed by type-III collagen fibers, represented as greenishcollagenous fibers. In the areas of loose connective tissue substitution, collagen type fibers are intercalated to a lesser extent by type-III collagen fibers. In longitudinal histological sections the type-I collagen fibers lose their linear arrangement. The disorganization of the tissue is well evidenced.

Chondroid metaplasia of the tendinous tissue in the MT-stained demonstrated a perfect formation of chondrocytes submerged in the abundant loose connective tissue stained in blue. The same areas in AB-stained samples formed a myxoid-lyke matrix, characterized by positive interlaced $\mathrm{AB}$-positive tissue bundles interspersed by chondrocytes. In PR-stained samples the polarization was restricted, tissue disorganization was composed of loose connective tissue and some type-III collagen fibers is observed intermingle of type-I collagen fibers.

Collagenolysis was associated with neovascularization and characterized by abundance of blood vessels between the loose connective tissue, which strongly stained in blue in the MT. In AB-stained samples, areas of loose connective tissue correspond to areas of neovascularized AB-positive matrix. In PRstained samples, polarization of most fibers occurs due to the lack of linear arrangement of the fibers composed of type-I collagen fibers, which have a smaller number of type-III fibers.

Lymphohistioplasmacytic tendinitis was characterized in the MT-stained samples by abundant loose connective tissue interspersed with dense connective tissue. In AB-stained samples the presence of chondrocytes wrapped by an AB-positive matrix. In the PR-stained samples, the polarization of the loose connective tissue occurred, most of the type I collagen fibers being accompanied by a lack of fascicular arrangement.

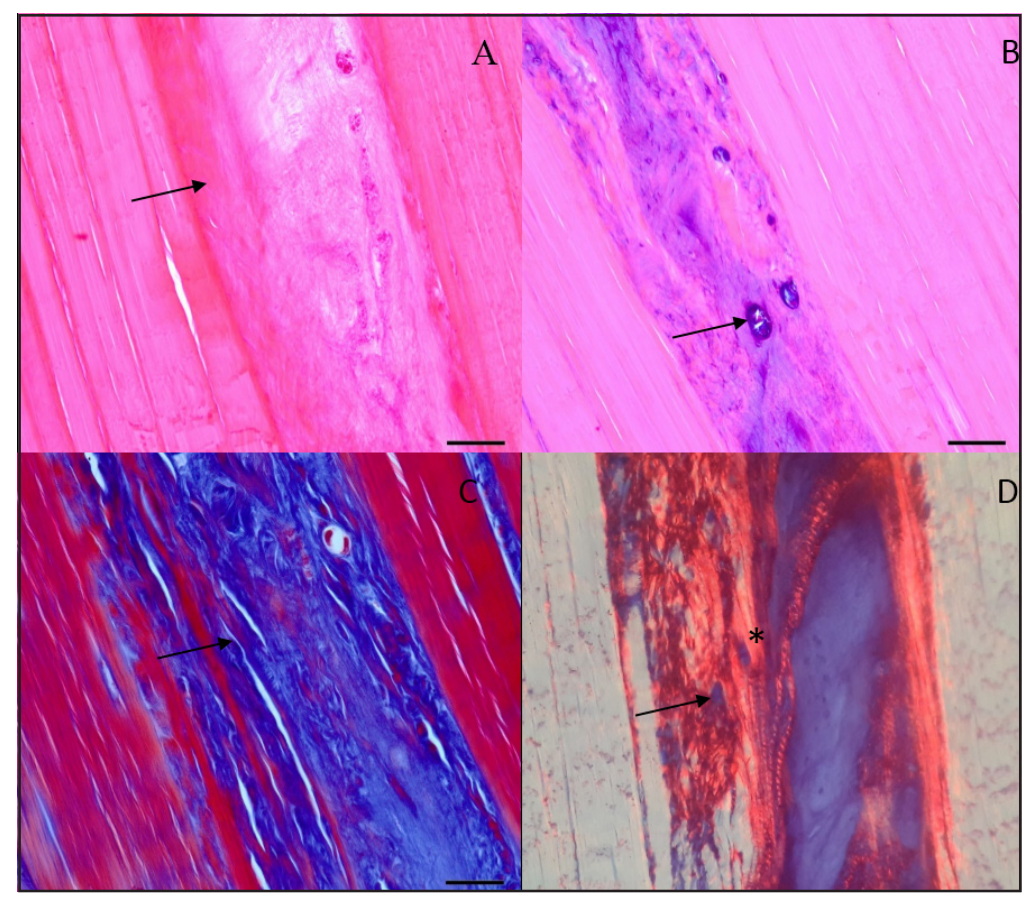

Figure 1. DDFT longitudinal section of the palmar aspect of the metacarpophalangeal joint. A- Fibroplasia and extensive multifocal cartilaginous metaplasia (arrow) [H\&E, 100x]. B- Chondrocytes enveloped by a positive matrix stained in blue (arrow) [Alcian blue stain, 100x]. C- Disorderly of loose connective tissue stained in blue (arrow) [Masson's trichrome stain, 100x]. D- Collagen fibers type I in stained in orange (asterisk) intercalated by collagen fibers type III stained in dark green under polarized light (arrow) [Picrosirius red stain, 100x]. 
Superficial Digital Flexor Tendon (SDFT) and junction of Manica Flexoria

Gross transversal sections of the SDFT showed lesions characterized by the presence of dark-yellow necrotic zones with soft consistency at the touch. The H\&E findings corresponded to lymphohistioplasmacytic inflammation and focally extensive severe fibrosis associated with condroid metaplasia in the epitenon region. Lymphohistioplasmacytic tendinitis was associated with necrotic, hemorrhagic and chondroid metaplasia areas, corresponding in the MT technique to a disorganized loose bluish connective tissue with numerous chondrocytes. The chondroid metaplasia was confirmed in the $\mathrm{AB}$ staining and was characterized by chondrocytes surrounded by an chondroid AB-positive matrix. In the PR stain, the areas composed by disorganized loose connective tissue corresponded to collagen type I fibers (orange polarized stain) intermingled with collagen type III fibers (green polarized stain). Grossly, manica flexoria in one tissue sample, at the junction of SDFT, presented pearly white tissue and in another sample there was thickened fibrous tissue, corresponding to collagenolysis (Figure 2-A) and chondroid metaplasia, respectively. The collagenolysis lesions associated with condroid metaplasia were characterized by loose connective tissue areas with interspersed dense connective tissue in MT staining (Figure 2-C). Abundant neovascularization associated with chondrocytes was seen. The loose connective tissue areas which by $\mathrm{AB}$ staining corresponded to a loose matrix stained in blue (Figure 2-B). Most type I collagen fibers polarized in PR stain associated to the presence of the dense connective tissue (Figure 2-D). In contrast, in the areas corresponded to loose connective tissue, a better polarization occurs and collagen fibers type I and III were observed in PR stained sections.

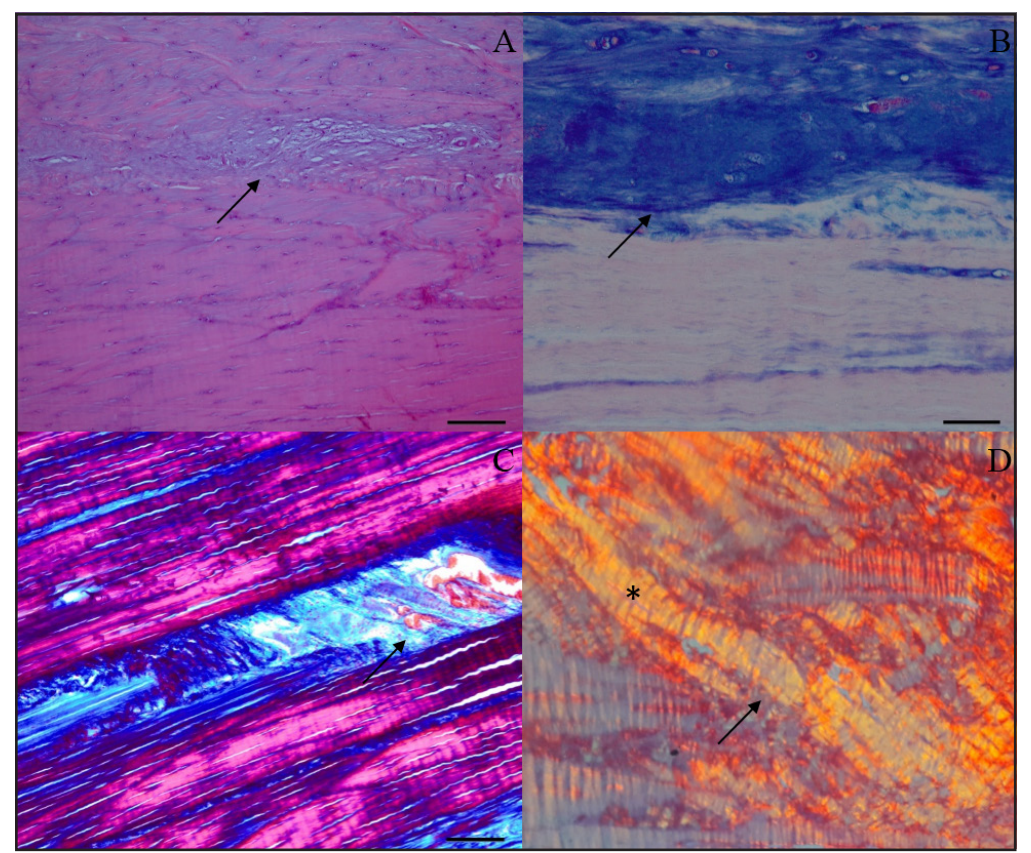

Figure 2. SDFT longitudinal section and junction of Manica Flexoria of the palmar aspect of the metacarpophalangeal joint and junction. A- Collagenolysis (arrow) [H\&E, 100x]. B- Positive matrix and presence of chondrocytes (arrow) [Alcian blue stain, 200x]. C- Disorderly of loose connective tissue stained in blue (arrow) [Masson's trichrome stain, 100x] D- Loss of linear arrangement of orange type I collagen fibers (arrow) interspersed with dark green type III collagen fibers under polarized light (asterisk) [Picrosirius red stain, 100x].

\section{Palmar Annular Ligament (PAL)}

Gross longitudinal lesions of the PAL were represented by thickened fibrous areas associated with a yellow discoloration and adhesions to the SDFT. There is multifocal fibrosis, probably in the adhesion area, with abundant neovascularization. Also, observed collagenolysis areas intermingled with chondrocytes in $H \& E$ were observed associated with a loss of collagen fibers linear arrangement, interspersed with neovascularized loose connective tissue. In the MTstained samples, the loose connective tissue appeared strongly stained in blue, interspersed by some areas of 
dense connective tissue stained in red, associated to neovascularization areas. In the PR-stained samples, polarization of the majority of the collagen fibers occurred; the lack of their linear arrangement was characterized by type-I collagen (thick orange fibers), in the middle of which a smaller type-III fibers (fine green fibers) were observed.

\section{Suspensory ligament (SL) lateral branch}

Gross transversal lesions of the SL lateral branch showed white fibrous tissue located in the center of the ligament and thickening of the epitenon region, associated with severe irregularity of the abaxial aspect of the proximal lateral sesamoid bone (insertion area). These H\&E-stained findings were characterized by the formation of islands of chondrocytes in the center of the fascicles associated with a thickened epitenon region. The fibrous tissue grossly corresponded to chondroid metaplasia, observed in $\mathrm{AB}$-stained as the numerous chondrocyteswere surrounded by highly AB-positive matrix interspersed in the ligament fibers and connective tissue.
Articular cartilage (AC) of metacarpus III condyle (MCIII)

Macroscopically, there was loss of AC of MCIII medial and lateral aspect of the condyle with exposure of the subchondral bone associated with a dark red color and clear presence of deep linear grooves on the surface. In the H\&E technique, it was observed a severe cartilage fibrillation and eburnation. In the two other cases, gross findings were characterized by a light-red color with strong and soft linear grooves in the MCIII condyle. In H\&E stain, it was observed a focally severe-to-moderate fibrillation (Figure 3-A). In the AB-stained samples, the surface layer and the matrix zone of calcified cartilage were weakly stained in blue. Articular cartilage with moderate changes had a marked decrease in staining in the superficial zone and the upper portion of the middle zone (Figure 3-B). In the severe changes, the marked blue $\mathrm{AB}$-stained matrix appeared only in the deep layers of the (Figure $3 \mathrm{C}-\mathrm{D})$. The normal AC is characterized by the three chondrocyte layers with different shapes, stained in strong blue in the $\mathrm{AB}$ staining technique.

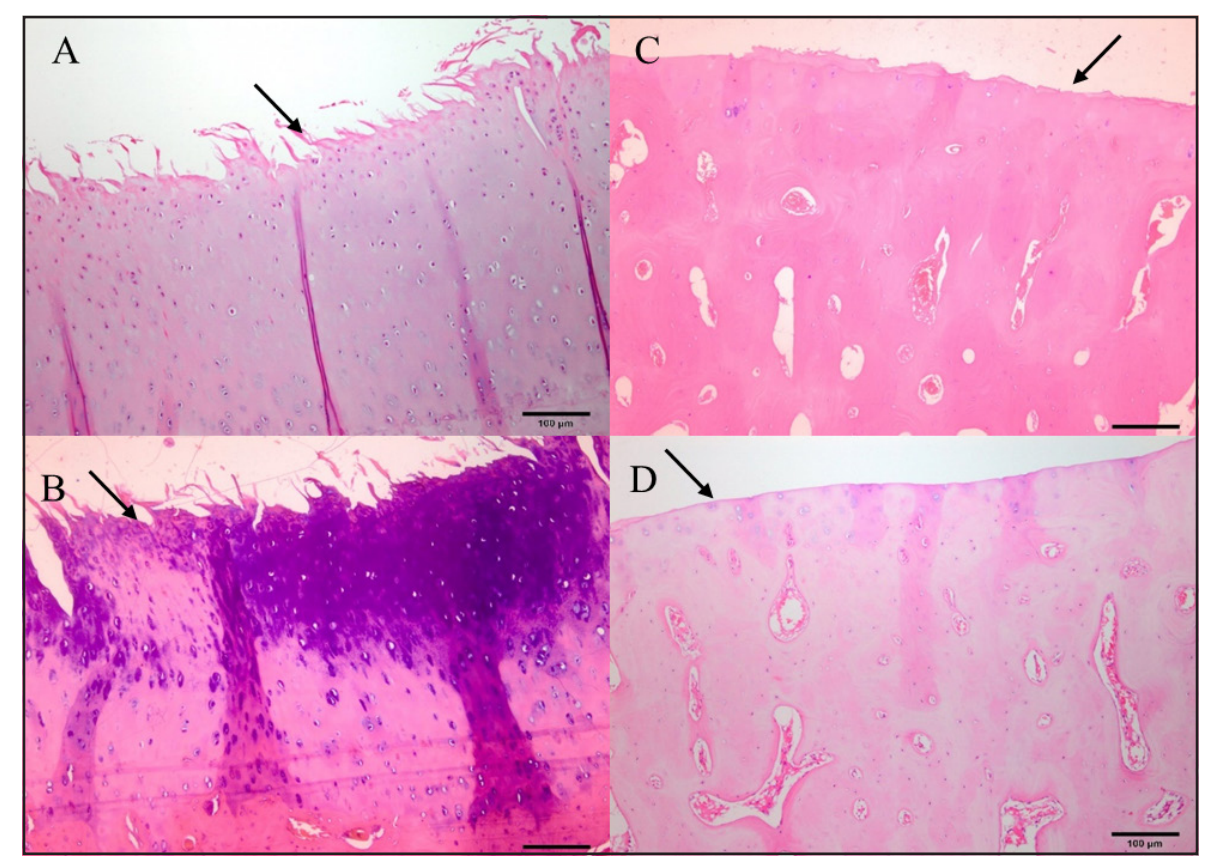

Figure 3. Articular Cartilage longitudinal section of the MCIII condyle. A- Fibrillation of the articular cartilage (arrow) [H\&E, 100x]. B- Decrease of blue stained in fibrillation of articular cartilage (arrow) [Alcian blue stain, 100x]. C- Eburnation of articular cartilage (arrow) [H\&E, 100x]. D- Severe decrease of blue stained in eburnation of articular cartilage (arrow) [Alcian blue stain, 100x].

\section{DISCUSSION}

Three special stains were utilized in this study along with the H\&E evaluation. Collagen fibers got stained intensively in most of staining techniques used in this study, especially in MT. Furthermore, different types of collagen fibers are not distinguished by MT stain alone. In contrary, the PR stain contributed to the study of collagen-made structures due to enhancement 
of the existing birefringence in the collagen, due to its parallelism and tissue organization [17]. Elastic fibers of collagen type I are usually characterized by thick fibers, highly birefringent and yellow or red colored in PR stain. In the other hand, type-III collagen of the reticular fibers usually has the smallest fibers with low birefringence and green color [20]. In this study, the normal matrix tendon was composed by dense connective tissue, stained in red by MT [16] and rich in collagen type I fibers by PR staining.

The identification of chondroid metaplasia and multifocal fibrosis in the ligaments were important findings in this study, and were associated with abundant loose connective tissue, and predominantly typeIII collagen fibers. Affections of the SL lateral branch contained white fibrous tissue [9] and, this finding was observed in this study. It has been described that fibrocartilaginous tissue of ligament specimens contained a few small hyaline cartilage-like areas, which were characterized by homogeneous basophilic matrix with scattered plump chondroid cells [27].

Fibroplasia in tendons are represented by increased fibroblasts cells and irregularities of the tissue organization in comparison to the normal tendon [19]. The important observation in this study was fibroplasia in tendons and ligaments, seems to be composed by abundant loose connective tissue, chondrocytes and intermingled collagen type I and III fibers associated with lack of crimps alignment of the fibers. The loose connective tissue is a disorganized tissue in the MTstained samples. The characteristic blue discoloration of collagen fibers was only observed in the loose connective tissue may be because the dye penetration becomes easier when compared to the dense connective tissue (stained in red). Immature collagen fibers and the endotendon stain in blue when using the MT technique [26]. The loose connective tissue appears to be less compact and soft. In areas of dense connective tissue substitution for loose connective tissue there is a better polarization of the collagen fibers observed in the PR stain, which is due to a greater fragility of the loose connective tissue. This fibrous repair is functionally deficient, weaker than the normal tendon in many ways. The natural repair mechanisms triggered by tendon damage often leads to the formation of biomechanically inferior scar tissues that is prone to re-injury [1]. The fragile structure suggested by the MT stain results (presence of the loose connective tissue) in this study perhaps make the tendons and ligaments receptive to other lesions.

Changes in the DDFT and SDFT evaluated in this study were characterized by hemorrhagic infiltration in the fascicles corresponding to necrotic zones and thickening of the epitenon. These findings corresponded to lymphohistioplasmacytic inflammatory infiltration and fibroplasia in the histologic evaluation, associated with deposition of loose connective tissue in the epitenon and lack of type-I collagen fibers parallelism intermingled with the deposition in type-III collagen fibers in reparation zones. This lymphohistioplasmacytic infiltration in tendons was previously described [5]. Specific tendon lesions should be considered as being result of vascular thrombosis and ischemia [4]. Remodeling of the initial repair process of the ligaments and tendons is composed by hemorrhagic, vascular congestion and necrotic areas [2]. In human chronic Achilles tendinopathy, neovascularization was one of the features associated with extracellular matrix degeneration [3,22]. In this study, collagenolysis areas intermingled with chondrocytes were observed associated with a loss of collagen fibers linear arrangement in the DDFT interspersed with neovascularized loose connective tissue.

Articular cartilage damage begins with the release of cytokines that triggers the degradation cascade of collagen in the cartilaginous matrix. With the progress of collagen depletion, cartilage loses its ability to absorb impact, and continuous exercise results in constant trauma and consequently cartilage insults. Synovial changes are the result of inflammation including hyperplasia and fibrosis. Bone changes follow in these cases and are characterized by the formation of peri-articular osteophytes, decreased joint space and sclerosis in the subchondral bone [24]. In one study, the process of osteoarthritis was characterized microscopically by the loss of proteoglycans (reduction of metachromatic staining by Toluidine blue) caused by the enzymatic degradation of articular cartilage (mainly by aggrecanase and metalloproteinases). Later on the process, it can be seen fragmented collagen fibers. Exposure and calcification of the cartilaginous layers are also observed, with consequent eburnation of the subchondral bone surface [14]. The Toluidine blue stain is usually used in the histological analysis to demonstrate the presence of glycosaminoglycans in the extracellular cartilaginous matrix, staining in light blue and dark purple (metochromasia) [11]. The samples of 
the articular cartilage degeneration obtained from the femoral lateral trochlear presented weak marking (light blue) and there was no metachromatic in Toluidine blue staining [23]. Toluidine blue was not performed in this study. Histologic examination in this study of AC by $\mathrm{AB}$ stain demonstrates the decrease of blue staining in lesions such as fibrillation of the AC, but the cause of this change was not determined.

\section{CONCLUSION}

The use of several histochemical techniques has shown that MT stain is an excellent technique to be used in tendons and ligaments, because it allowed distinguishing the presence of loose connective tissue from dense connective tissue. As fibroplasia and collagenolysis (observed in H\&E sections) evidenced the weakness of the substitution of the dense for loose connective tissue, the MT and PR allowed to better identify the nature of these substitution connective tissue.

\section{MANUFACTURER}

${ }^{1}$ EasyPath Kit. São Paulo, SP, Brazil.

Declaration of interest. The authors report no conflicts of interest. The authors alone are 402 responsible for the content and writing of the paper.

\section{REFERENCES}

1 Barsby T., Bavin E.P. \& Guest D.J. 2014. Three Dimensional Culture and Transforming Growth Factor beta 3 Synergistically Promote Tenogenic Differentiation of Equine Embryo-Derived Stem Cells. Tissue Engineering. 20: 26042613.

2 Barreira A.P.B. 2005. Implante autólogo de células mesenquimais no tratamento de tendinites induzida em equinos: avaliação clínica, ultra-sonográfica e imunoistoquímica. 98f. Botucatu, SP. Tese (Doutorado em Medicina Veterinária) - Faculdade de Medicina Veterinária e Zootecnia, Universidade Estadual Paulista "Júlio Mesquita Filho.

3 Beck S., Blunden T., Dyson S. \& Murray R. 2011. Are matrix and vascular changes involved in the pathogenesis of deep digital flexor tendon injury in the horse? Veterinary Journal. 189: 289-295.

4 Busoni V., Heimann M., Trenteseaux J., Snaps F. \& Dondelinger R.F. 2005. Magnetic resonance imaging findings in the equine deep digital flexor tendon and distal sesamoid bone in advanced navicular disease - an ex vivo study. Veterinary Radiology Ultrasound. 46: 279-286.

5 Carvalho, A.M., Alves A.L.G., Oliveira P.G.G., Álvarez L.E.C., Amorim R.L., Hussni C.A. \& Deffune E. 2011. Use of adipose tissue-derived mesenchymal stem cells for experimental tendinitis therapy in equines. Journal of Equine Veterinary Science. 31: 26-34.

6 Culling C.F.A., Allison R.T. \& Bars W.T. 1985. Cellular pathology technique. 4th edn. London: Butterworths, 232p.

7 De Bastiani G., De La Côrte F.D., Brass K.E., Kommers G.D. \& Denoix J.M. 2014. Association of ultrasound and anatomopathologic findings of equine metacarpophalangeal lesions. Journal Equine Veterinary Science. 34: 1218-1225.

8 Dyson S.J. 2004. Medical Management of superficial digital flexor tendonitis: a comparative study in 219 horses (1992-2000). Equine Veterinary Journal. 36: 415-419.

9 Halper J., Kim B., Khan A., Yoon J.H. \& Mueller P.O. 2006. Degenerative suspensory desmitis as a systemic disorder characterized by proteoglycan accumulation. BMC Veterinary Research. 12: 1-14.

10 Hayashi K., Manley P.A. \& Muir P. 2004. Cranial cruciate ligament pathophysiology in dogs with cruciate disease: a review. Journal of the American Animal Hospital Association. 40: 385-390.

11 Hoemann C., Kandel R., Roberts S., Saris D.B.F., Creemers L., Mainil-Varlet P., Méthot S., Hollander A.P. \& Buschmann M.D. 2011. International cartilage repair society (ICRS) recommend guidelines for histological end-points for cartilage repair studies in animal models and clinical trials. Cartilage. 2: 153-172.

12 Kannus P. 1977. Etiology and pathophysiology of chronic tendon disorders in sports. Scandinavian Journal of Medicine \& Science in Sports. 7: 78-85.

13 Kastelic J., Galeski A. \& Baer E. 1978. The multicomposite structure of the tendon. Connective Tissue Research. 6 : $11-23$.

14 Kidd J.A., Fuller C. \& Barr A.R.S. 2001. Osteoarthritis in the horse. Equine Veterinary Education. 13: 160-168.

15 Liu C.F. Aschbacher-Smith L., Barthelery N.J., Dyment N., Butler D. \& Wylie C. 2011. What We Should Know Before Using Tissue Engineering Techniques to Repair Injured Tendons: A Developmental Biology Perspective. Tissue Engineering Part B-Reviews. 17: 165-176. 
16 Mcbride D.J., Hahn R.A. \& Silver F.H. 1985. Morphological characterization of tendon development during chick embryogenesis: measurement of birefringence retardation. International Journal of Biological Macromolecules. 7: 71-76.

17 Montes G.S. 1996. Structural biology of the fibers of the collagenous and elastic systems. Cell Biology International. 20: $15-27$.

18 Mow V.C. \& Ratcliffe A. 1997. Structure and function of articular cartilage and meniscus. In: Mow V.C. \& Hayes W.C. (Eds). Basic Orthopaedic Biomechanics. 2nd edn. Philadelphia: Lippincott-Raven, pp.113-177.

19 Oliveira P.G.G., Alves A.L.G., Carvalho A.M., Hussni C.A., Watanable M.J., Amorim R.L., Rodrigues M.M.P. \& Mota L.S. 2011. Uso de células mononucleares da medula óssea no tratamento de tendinites induzidas experimentalmente em equinos. Arquivo Brasileiro de Medicina Veterinária e Zootecnia. 63: 1391-1398.

20 Ortega H.H., Muñoz-de-Toro M.M., Luque E.H. \& Montes G.S. 2003. Morphological characteristics of the interpubic joint (Symphysis pubica) of rats, guinea pigs and mice in different physiological situations - a comparative study. Cells Tissues Organs. 173: 105-144.

21 Pennisi E. 2002. Tending tender tendons. Science. 295: 1011.

22 Petersen W., Pufe T., Zantop T., Tillmann B., Tsokos M. \& Mentlein R. 2004. Expression of VEGFR-1 and VEGFR-2 in degenerative Achilles tendon. Clinical Orthopaedics and Related Research. 420: 286-291.

23 Rocha J. 2016. Avaliações macroscópica e histológica do reparo da cartilagem articular equina tratada com microperfurações do osso subcondral associadas ou não a injeção intra-articular de cartogenina. Pequisa Veterinária Brasileira. 36: $272-278$.

24 Santschi E.M. 2008. Articular fetlock injuries in exercising. Veterinary Clinics of North America: Equine Practice. 24: $117-132$.

25 Satomi E., Teodoro W.R., Parra E.R., Fernandes T.D., Velosa A.P.P., Capelozzi V.L. \& Yoshinaria N.H. 2008. Changes in histoanatomical distribuition of types I, III and V collagen promote adaptative remodeling in posterior tibial tendon rupture. Clinical Science. 63: 9-14.

26 Silva R.M.G. 2007. Estudo morfometrico, ultra-estrutural e imuno-histoquimico do ligamento cruzado cranial com ruptura em cães. 126f. São Paulo, SP. Tese (Doutorado em Ciências) - Faculdade de Medicina Veterinária e Zootecnia, Universidade de São Paulo.

27 Smith R. 2013. Diagnosis and management of soft tissue injuries in the fetlock region of athletic horses. In: Proceedings of the 1st Brasil Horse Center Laboratório e Clínica Veterinária Conference (Rio de Janeiro, Brasil). pp.73-77.

28 Webbon P.M. 1977. A postmortem study of equine digital flexors tendons. Equine Veterinary Journal. 9: 61-67. 